\title{
Determination of the Influence of the Antiphlogistic Ingredients Panthenol and Bisabolol on the SPF Value in vivo
}

\author{
Milena Werner ${ }^{a} \quad$ Marianne Herling ${ }^{a} \quad$ Birgit Garbe $^{a} \quad$ Carmen Theek $^{b}$ \\ Hagen Tronnier ${ }^{a} \quad$ Ulrike Heinrich ${ }^{a}$ Nicole Braun $^{a}$ \\ ${ }^{a}$ DermaTronnier GmbH \& Co. KG, Institute for Experimental Dermatology, Witten/Herdecke University, and \\ ${ }^{b}$ Carmen Theek, CTS, Witten, Germany
}

\section{Keywords}

Sunscreen · Antiphlogistic ingredients · Panthenol .

Bisabolol · Sun protection factor · Ultraviolet irradiation

\begin{abstract}
Background: Today, the sun protection factor (SPF) value of sunscreen products is determined in vivo with a standardized protocol (EN ISO 24444:2010), and the measured SPF biological end point is the visible skin erythema. However, many of the sunscreen products currently available on the market have antiphlogistic ingredients, which may potentially result in an overestimated SPF of the sunscreen. Aims: To investigate the potential influence of the antiphlogistic ingredients panthenol and bisabolol in sunscreens on the determined SPF value in vivo. Methods: Formulations with different concentrations of the antiphlogistic ingredients bisabolol or panthenol were tested. As a reference, a base formulation (vehicle) without antiphlogistic ingredients was used. First, the SPF of the sunscreen formulas with and without antiphlogistic ingredients was analyzed in vitro. To investigate whether the antiphlogistic ingredient may suppress the inflammatory response to ultraviolet (UV) irradiation, the SPF was determined in vivo. Finally, selected formulations were also analyzed in an erythema model for
\end{abstract}

\section{KARGER}

(C) 2017 S. Karger AG, Basel

E-Mail karger@karger.com

www.karger.com/spp testing formulations on UV-induced inflammation. Results: It could be confirmed that no differences between the formula with and that without the active antiphlogistic ingredients bisabolol or panthenol exist when measured in vitro. However, there was also no statistically significant difference in the erythemal response between the vehicle (without an antiphlogistic active ingredient) and the test formulations with different concentrations of the antiphlogistic active ingredients in the in vivo determination of the SPF. Evidence of anti-inflammatory activity of the sunscreen antiphlogistics bisabolol and panthenol was also not apparent in the UV model over a time course of 48 h. Conlusion: The antiphlogistic ingredients panthenol and bisabolol incorporated in the tested sunscreen formula do not interfere with erythema reddening and thus do not affect the SPF value in vivo.

(C) 2017 S. Karger AG, Basel

\section{Introduction}

Sunscreen products aim to help protect the skin against ultraviolet (UV) rays and consequently reduce the risk of early skin aging and skin cancer. They are classified as a "cosmetic product" and not as a "medical product" according to Regulation (EC) No. 1223/2009 [1]. Further-

Dr. Nicole Braun

DermaTronnier, Institute for Experimental Dermatology at the Witten/Herdecke University

Alfred-Herrhausen-Strasse 44, DE-58455 Witten (Germany)

E-Mail nicole.braun@uni-wh.de 
more, criteria for cosmetic product labeling and for special claims are published in the Guidelines to Regulation No. 655/2013 [2], where it is stated that it is not allowed to claim special characteristics for a cosmetic product without scientific evidence. The sun protection factor (SPF) sunscreen labels in the European Union are divided into 4 categories, namely low (SPF 6 and 10), medium (SPF 15, 20, and 25), high (SPF 30 and 50), and very high (SPF 50+). The SPF is quantified with a standardized testing method according to EN ISO 24444:2010 [3]. In short, the minimum erythemal dose (MED) on unprotected and on sunscreen-protected skin is measured and the justperceptible visible sunburn (redness) is used as a biological end point.

However, sunscreens not only protect against UV rays and their consequential damages, it is also claimed that they offer a number of further benefits for the skin. This includes, for example, formulations with humectants such as glycerin and urea, tanning accelerators, insect repellents, and antioxidants. Recently, sunscreens have been formulated with antiphlogistic ingredients to help alleviate pain, redness, and burning. The main ingredients with soothing and anti-inflammatory effects are bisabolol, panthenol, allantoin, and aloe.

Natural bisabolol is a major component of German chamomile as well as yarrow. In vitro and in vivo studies have demonstrated the anti-inflammatory effect of bisabolol, i.e., the suppression of proinflammatory cytokine production [4]. Furthermore, it has antiseptic, soothing, antibiotic, fungicidal, and anticarcinogenic properties.

Panthenol is also used in various formulations in cosmetics. In the skin, it is oxidized to pantothenic acid, which, in turn, is required to synthesize coenzyme A (CoA). CoA plays an essential role in a variety of biochemical reactions. Panthenol has been shown to improve skin hydration and reduce transepidermal water loss. In addition, the proliferation of fibroblasts is stimulated, which has a positive effect on wound healing.

However, the use of antiphlogistic ingredients in sunscreen products is questionable as they may cause a decrease in erythema, which might lead to an overestimation of the SPF value. In this case, cellular and genomic damage by UV radiation occurs without signs of erythema, leading customers to believe in a high level of protection.

For this reason, in 2013, Haydar and Burkhart [5] requested the FDA Commissioner to caution manufacturers to not use anti-inflammatory ingredients in their sunscreen products until more research has been conducted on the potential harm of these additives.

Influence of Antiphlogistic Ingredients on the SPF
In addition, in March 2013, Sayre [6] submitted a citizen petition to the FDA, requesting the withdrawal of the approval of the anti-inflammatory sunscreen filter ingredients dioxybenzone, oxybenzone, trolamine salicylate, homosalate, and octisalate.

Due to the high relevance of this topic, the effect of the antiphlogistic ingredients panthenol and bisabolol on the SPF value in vivo on early and delayed erythema response has been investigated here.

\section{Methods}

\section{Materials}

Two sunscreen formulas with different concentrations of panthenol and bisabolol were prepared. In line A, the concentrations correlated with those of commercial sunscreen products; in line B, they were slightly higher than in sunscreen products available on the market.

Line A. A sunscreen formula with an estimated SPF value of 20 was prepared in 5 variations. In detail, 1 variation contained no antiphlogistic ingredient (vehicle A), 2 variations contained the active ingredient panthenol in concentrations of 0.6 and $0.75 \%$, respectively, and 2 others contained the active ingredient bisabolol in concentrations of 0.1 and $0.2 \%$, respectively (Table 1 ).

Line B. A further sunscreen formula with an estimated SPF value of 15 was prepared in 3 variations. In detail, 1 variation contained no antiphlogistic ingredient (vehicle B), 1 contained the active ingredient $\mathrm{D}$-panthenol in a concentration of $1 \%$, and 1 contained the active ingredient bisabolol in a concentration of $1 \%$ (Table 2).

We used standard S2 (Institut Dr. Schrader Ancopharm, Holzminden, Germany) as the reference for the SPF determination in vitro, the P3 high SPF reference sunscreen standard (Institut Dr. Schrader Ancopharm) as the reference for the SPF determination in vivo, and $1 \%$ hydrocortisone cream (Hexal AG, Holzkirchen, Germany) as a positive control in the UV model.

\section{Study I: SPF Determination in vitro}

Polymethyl methacrylate (PMMA) slides (Schönberg GmbH \& Co. KG, Hamburg, Germany) with a surface area of $25 \mathrm{~cm}^{2}$ and a roughness of $6 \mu \mathrm{m}$ were used for the SPF in vitro determination. The PMMA plates were evenly coated with $1.3 \mathrm{mg} / \mathrm{cm}^{2}$ of the different sunscreen formulas and incubated for $15 \mathrm{~min}$ at $30^{\circ} \mathrm{C}$. The coated plates were preirradiated on 6 spots with 1 MED with UV light (UVA + UVB) in the range of 290-400 nm (Multiport Solar Simulator, Solar Light Company, Inc., Philadelphia, PA, USA). One MED corresponds to $250 \mathrm{~J} / \mathrm{m}^{2}$ for skin type II (reference skin type) weighted against the erythema action spectrum [7]. The accurate measurement of UVB radiation was performed by the erythma detector PMA2108 LLG (Solar Light Company, Inc.). The measurements and data analysis of the samples were conducted with the UV-2000S device Labsphere 2000S (Labsphere Inc., North Sutton, NH, USA) before and after irradiation. A total of 5 plates per formulation were used, and 6 measurements on each plate were performed. Therefore, each result is the mean of 30 measurements. 
Table 1. International Nomenclature of Cosmetic Ingredients declaration of vehicle $\mathrm{A}$

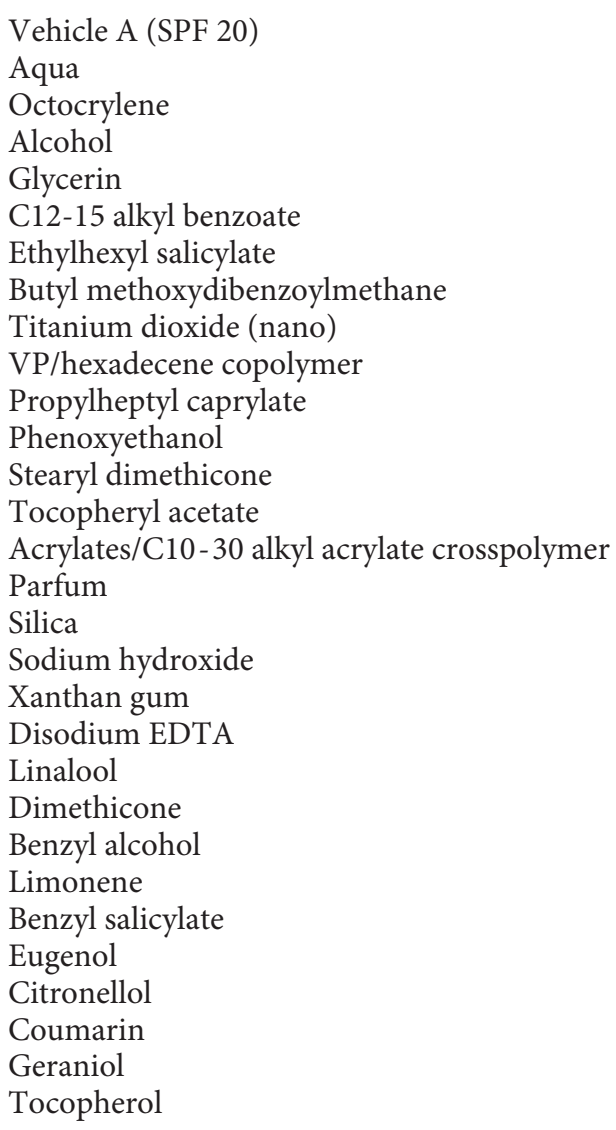

\section{Test Subjects}

Healthy skin phototype I-III [8] test subjects aged 18-70 years participated in this study. Inclusion criteria were a healthy skin, normal nutritional habits, and a BMI of 18-25. Exclusion criteria were pregnancy, breast-feeding, smoking, medications which affect the skin response such as antibiotics, anti-inflammatory medications, skin diseases, photosensitivity, metabolic diseases, sunbathing or the use of sunbeds, and skin moles in the test area. The subjects read the information sheets and signed the informed consent form prior to study participation. The study design for noninvasive surveys was approved by the Ethics Committee of the Witten/Herdecke University, Witten, Germany.

\section{Study II: SPF Determination in vivo}

The SPF values were determined according to the international standard EN ISO 24444:2010. In detail, 30 test subjects with phototypes I-III according to Fitzpatrick [8] or an ITA value $>28^{\circ}$ by colorimetric methods were selected and randomly assigned to 1 of 3 groups [9] (Table 3). Individual, just-perceptible MED was determined on the previously unexposed back of the test subjects using different light intensities at $12 \%$ increments. The UV source was the same as in study I. The sunscreen formula (an amount of
Table 2. International Nomenclature of Cosmetic Ingredients declaration of vehicle $B$

Vehicle B (SPF 15)

Aqua

Dibutyl adipate

Cocoglycerides

Coco-caprylate

Diethylamino hydroxybenzoyl hexyl benzoate

Glycerin

Ethylhexyl triazone

Cetearayl alcohol

Disodium cetearyl sulfosuccinate

Bis-ethylhexyloxyphenol methoxyphenyl triazine

Phenoxyethanol

Soidum polyacrylate

Xanthan gum

Dinatrium EDTA
$2 \mathrm{mg} / \mathrm{cm}^{2}$ ) was applied carefully on the back of each test subject, and followed by UV irradiation after $20 \mathrm{~min}$. This was followed by an examination of the skin's response, in the form of erythema, 16-24 h after UV exposure.

\section{Study III: The UV Model}

The UV model has proven to be a useful method for testing the antiphlogistic effect of a topically applied substance. Twelve test subjects with phototypes I-III according to Fitzpatrick [8] or an ITA value $>28^{\circ}$ by colorimetric methods were selected [9] (Table 3). First, the individual erythema threshold was determined for each subject on the previously unexposed back using different light intensities at $25 \%$ increments. Test areas were then irradiated with a 1.25 MED. The UV source was the same as in studies I and II. The sunscreen formulas as well as $1 \%$ hydrocortisone cream (as a standard anti-inflammatory agent) were applied immediately afterwards on the irradiated areas. Since erythema only begins to develop 2-3 h after UV irradiation and reaches a maximum after $16-24 \mathrm{~h}$, the first measurements were performed after $6 \mathrm{~h}$, and then repeated after 24 and $48 \mathrm{~h}$. At indicated time points/intervals, the developing erythema was evaluated by photometric assessment using a chromameter (CR-400, Konika Minolta, Marunouchi, Japan), and by blood flow assessment using laser Doppler flowmetry (O2C device, Lea Medizintechnik GmbH, Giessen, Germany), followed by further applications of the sunscreen formula or $1 \%$ hydrocortisone cream. After UV irradiation, it is expected that the $\mathrm{a}^{*}$-value (skin redness) and blood flow at a depth of $1 \mathrm{~mm}$ will be higher than the baseline readings for indicating erythema. To be effective as an anti-inflammatory agent in the sunscreen formula, the $a^{*}$-value and blood flow should decrease after the treatment when compared to the vehicle formula.

\section{Statistical Analysis}

Descriptive summary statistics including mean, median, standard deviation (SD), and 95\% confidence interval (CI) were determined. In order to consider the course of parameters ( $a^{*}$-value and blood flow) over time, the area under the curve (AUC) was calcu- 
Table 3. Test subjects' characteristics at inclusion for the SPF in vivo and antiphlogistic efficacy testing

\begin{tabular}{|c|c|c|c|c|}
\hline & \multicolumn{3}{|c|}{ SFP in vivo determination } & \multirow[t]{2}{*}{ UV model } \\
\hline & group 1 & group 2 & group 3 & \\
\hline Test subjects, $n$ & 10 & 10 & 10 & 12 \\
\hline Products tested & $\begin{array}{l}\text { Vehicle A } \\
+0.6 \% \text { panthenol } \\
+0.75 \% \text { panthenol } \\
\text { P3 }\end{array}$ & $\begin{array}{l}\text { Vehicle A } \\
+0.1 \% \text { bisabolol } \\
+0.2 \% \text { bisabolol } \\
\text { P3 }\end{array}$ & $\begin{array}{l}\text { Vehicle } \mathrm{B} \\
+1 \% \text { bisabolol } \\
+1 \% \text { panthenol } \\
\text { P3 }\end{array}$ & $\begin{array}{l}\text { Vehicle A } \\
+0.2 \% \text { bisabolol } \\
\text { Vehicle B } \\
+1 \% \text { bisabolol }\end{array}$ \\
\hline $\begin{array}{l}\text { Mean age, years } \\
\text { Males/females, } n \\
\text { Skin phototype (I/II/III), } n\end{array}$ & $\begin{array}{l}46 \\
2 / 8 \\
6 / 2 / 2\end{array}$ & $\begin{array}{l}52 \\
1 / 9 \\
4 / 3 / 3\end{array}$ & $\begin{array}{l}44 \\
5 / 5 \\
2 / 2 / 6\end{array}$ & $\begin{array}{l}44 \\
6 / 6 \\
3 / 4 / 5\end{array}$ \\
\hline
\end{tabular}

Table 4. Descriptive statistics for SPF in vitro test of the vehicle A formula and the respective formula with antiphlogistic ingredients

\begin{tabular}{|c|c|c|c|c|c|}
\hline & \multirow[t]{2}{*}{ Mean } & \multicolumn{2}{|c|}{$95 \% \mathrm{CI}$} & \multirow[t]{2}{*}{$\mathrm{SD}$} & \multirow[t]{2}{*}{ Median } \\
\hline & & $\begin{array}{l}\text { lower } \\
\text { limit }\end{array}$ & $\begin{array}{l}\text { upper } \\
\text { limit }\end{array}$ & & \\
\hline \multicolumn{6}{|l|}{ Vehicle A } \\
\hline Unirradiated & 35.2 & 33.0 & 37.4 & 5.9 & 37.0 \\
\hline Irriadiated & 34.3 & 32.2 & 36.5 & 5.7 & 35.9 \\
\hline Difference & -0.8 & -1.1 & -0.6 & 0.7 & -0.8 \\
\hline \multicolumn{6}{|l|}{$+0.6 \%$ panthenol } \\
\hline Unirradiated & 36.3 & 34.3 & 38.3 & 5.3 & 36.0 \\
\hline Irriadiated & 35.6 & 33.6 & 37.7 & 5.6 & 36.1 \\
\hline Difference & -0.6 & -1.1 & -0.2 & 1.2 & -0.8 \\
\hline \multicolumn{6}{|l|}{$+0.75 \%$ panthenol } \\
\hline Unirradiated & 33.3 & 31.2 & 35.5 & 5.8 & 32.7 \\
\hline Irriadiated & 32.6 & 30.4 & 34.8 & 5.8 & 32.4 \\
\hline Difference & -0.7 & -1.1 & -0.4 & 1.0 & -0.7 \\
\hline \multicolumn{6}{|l|}{$+0.1 \%$ bisabolol } \\
\hline Unirradiated & 36.4 & 33.7 & 39.2 & 7.4 & 35.9 \\
\hline Irriadiated & 34.4 & 31.7 & 37.2 & 7.4 & 34.7 \\
\hline Difference & -2.0 & -4.0 & 0.0 & 5.3 & -0.7 \\
\hline \multicolumn{6}{|l|}{$+0.2 \%$ bisabolol } \\
\hline Unirradiated & 35.7 & 33.6 & 37.7 & 5.6 & 34.3 \\
\hline Irriadiated & 34.8 & 32.7 & 36.8 & 5.4 & 33.3 \\
\hline Difference & -0.9 & -1.3 & -0.5 & 1.0 & -1.0 \\
\hline
\end{tabular}

lated. The AUC values were normalized to the untreated, nonirradiated control area (blank). The Wilcoxon signed-rank test for dependent samples or rank-sum test for independent samples was applied two-sided for comparisons within or between groups using an a level of 5\%. All statistical analyses were performed with SAS (SAS Institute, Cary, NC, USA) v9.3 and graphs were created with GraphPad Prism v6 (GraphPad Software, San Diego, CA, USA).

Influence of Antiphlogistic Ingredients on the SPF

\section{Results}

\section{SPF Determination in vitro}

Additional antiphlogistic ingredients may potentially result in an overestimation of the SPF in the sunscreen product. However, SPF in vitro methods do not take the antiphlogistic effect into consideration. Here, we first analyzed the SPF of both formulas with and without incorporating the antiphlogistic ingredients in vitro.

Before irradiation, the vehicle A formula (without antiphlogistic ingredients) had a 95\% CI of 33.0-37.4; after irradiation, it was $32.2-36.5$. The $95 \%$ CI of the formula containing panthenol and bisabolol was 31.2-39.2 before irradiation and 30.4-34.8 after irradiation (Table 4).

The vehicle B formula showed a 95\% CI of 16.7-18.9 before irradiation and 15.9-17.5 after irradiation. The formulation with D-panthenol and bisabolol had a $95 \%$ CI of 16.9-19.3 before irradiation and 15.9-17.9 after irradiation (Table 5 ).

In both the lines of sunscreen formulas, the 95\% CI values of the vehicle and the vehicle with an incorporated antiphlogistic ingredient were within a comparable range before and after irradiation. Thus, it could be confirmed that there are no differences in the SPF in vitro between the formulas with and without antiphlogistic ingredients.

\section{SPF Determination in vivo}

To determine whether the antiphlogistic ingredients bisabolol or panthenol in sunscreen formulas interfere with erythema reddening, the SPF in vivo values were measured with and without the antiphlogistic ingredients. 


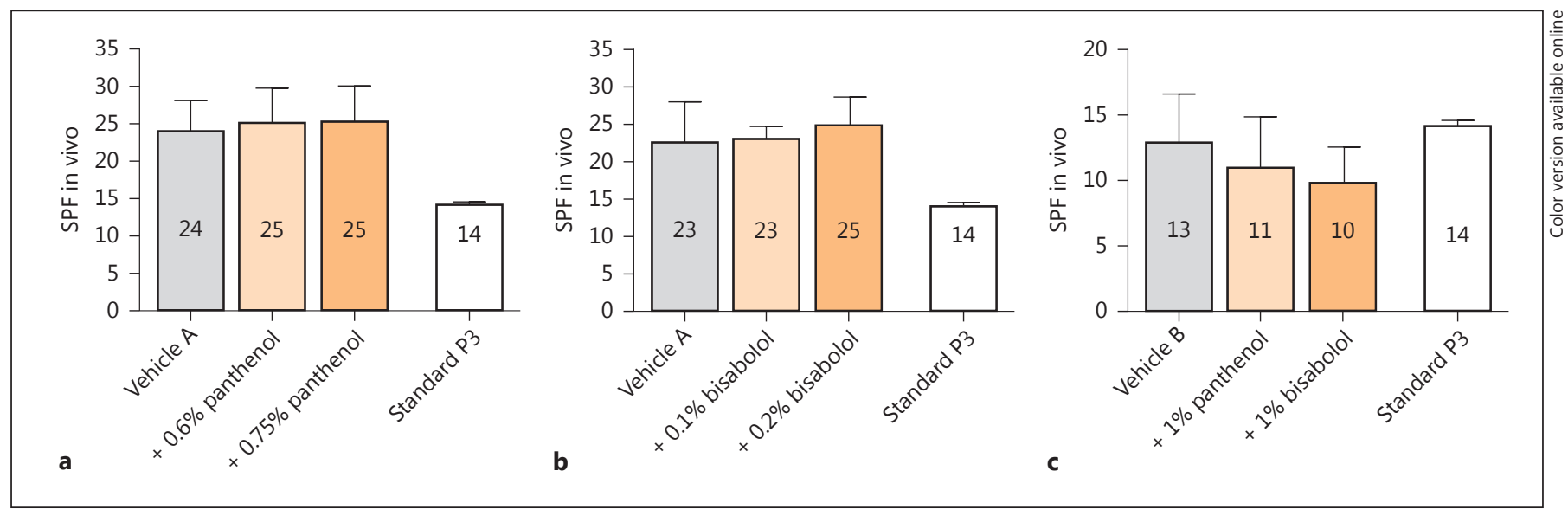

Fig. 1. a-c SPF determination in vivo. The bars indicate means \pm SD for 10 test subjects. The P3 high SPF reference sunscreen standard was used as a reference.

Table 5. Descriptive statistics for the SPF in vitro test of the vehicle $\mathrm{B}$ formula and the respective formula with antiphlogistic ingredients

\begin{tabular}{|c|c|c|c|c|c|}
\hline & \multirow[t]{2}{*}{ Mean } & \multicolumn{2}{|c|}{$95 \% \mathrm{CI}$} & \multirow[t]{2}{*}{$\mathrm{SD}$} & \multirow[t]{2}{*}{ Median } \\
\hline & & $\begin{array}{l}\text { lower } \\
\text { limit }\end{array}$ & $\begin{array}{l}\text { upper } \\
\text { limit }\end{array}$ & & \\
\hline \multicolumn{6}{|l|}{ Vehicle B } \\
\hline Unirradiated & 17.8 & 16.7 & 18.9 & 3.0 & 17.0 \\
\hline Irriadiated & 16.7 & 15.9 & 17.5 & 2.2 & 16.6 \\
\hline Difference & -1.1 & -1.5 & -0.7 & 1.1 & -0.8 \\
\hline \multicolumn{6}{|l|}{$+1 \%$ bisabolol } \\
\hline Unirradiated & 17.8 & 16.9 & 18.6 & 2.3 & 17.7 \\
\hline Irriadiated & 16.5 & 15.9 & 17.2 & 1.7 & 17.0 \\
\hline Difference & -1.2 & -1.5 & -1.0 & 0.7 & -1.1 \\
\hline \multicolumn{6}{|l|}{$+1 \%$ panthenol } \\
\hline Unirradiated & 18.5 & 17.6 & 19.3 & 2.3 & 18.4 \\
\hline Irriadiated & 17.3 & 16.7 & 17.9 & 1.6 & 17.4 \\
\hline Difference & -1.2 & -1.8 & -0.6 & 1.6 & -0.8 \\
\hline
\end{tabular}

In group 1, the value was found to be SPF $=24$ for the vehicle $\mathrm{A}$ formula. For the formula containing $0.6 \%$ or $0.7 \%$ panthenol, it was SPF $=25$ (Fig. 1a). In group 2, the value determined was $\mathrm{SPF}=23$ for the vehicle $\mathrm{A}$ formula. For the formula with $0.1 \%$ bisabolol, it was $\mathrm{SPF}=23$. For the formulation with $0.2 \%$ bisabolol, it was $\mathrm{SPF}=25$ (Fig. 1b). Thus, the formulas with antiphlogistic ingredients showed the same or marginally higher SPF values than the vehicle formula (Table 6).

The value found for the vehicle B formula was SPF = 13. For the formula with $1 \%$ bisabolol, it was $\mathrm{SPF}=10$.
Table 6. Statistical comparison of the vehicle formulas and their respective formulas with antiphlogistic ingredients

\begin{tabular}{lcccc}
\hline Comparison & Mean & SD & Median & $p$ value \\
\hline $\begin{array}{l}\text { Group 1 } \\
\text { Vehicle A }\end{array}$ & & & & \\
$\quad+0.6 \%$ panthenol & 1.12 & 4.45 & 0.00 & 0.5625 \\
$\quad+0.75 \%$ panthenol & 1.29 & 1.89 & 0.69 & 0.0938 \\
\hline $\begin{array}{l}\text { Group 2 } \\
\text { Vehicle A }\end{array}$ & & & & \\
$\quad+0.1 \%$ bisabolol & 0.31 & 5.35 & 0.00 & 0.9375 \\
$\quad+0.2 \%$ bisabolol & 2.17 & 6.34 & 1.73 & 0.3750 \\
\hline $\begin{array}{l}\text { Group 3 } \\
\text { Vehicle B }\end{array}$ & & & & \\
$\quad+1 \%$ bisabolol & -2.94 & 4.21 & -4.00 & 0.0840 \\
$\quad+1 \%$ panthenol & -1.63 & 2.64 & -1.55 & 0.0859 \\
\hline & & & & \\
\hline
\end{tabular}

For the formula with $1 \%$ D-panthenol, it was SPF $=11$ (Fig. 1c). So here, the formula with either bisabolol or Dpanthenol showed a slightly decreased SPF value compared to the vehicle formula. However, this difference was not statistically significant (Table 6).

The control product P3 high SPF reference sunscreen standard, which was tested at the same time, showed normal sensitivity for the tested group $(\mathrm{SPF}=14)$.

The results indicate that the incorporation of the antiphlogistic ingredients panthenol and bisabolol had no impact on the measured SPF in vivo. 
Fig. 2. a-d Antiphlogistic effect of $0.2 \%$ and $1 \%$ bisabolol evaluated by redness ( $\mathrm{a}^{*}$-value) and blood flow at a depth of $1 \mathrm{~mm}$, in comparison to the vehicle formulas, irradiated control (skin was irradiated and did not receive any topical treatment), and $1 \%$ hydrocortisone cream.

Influence of Antiphlogistic Ingredients on the SPF
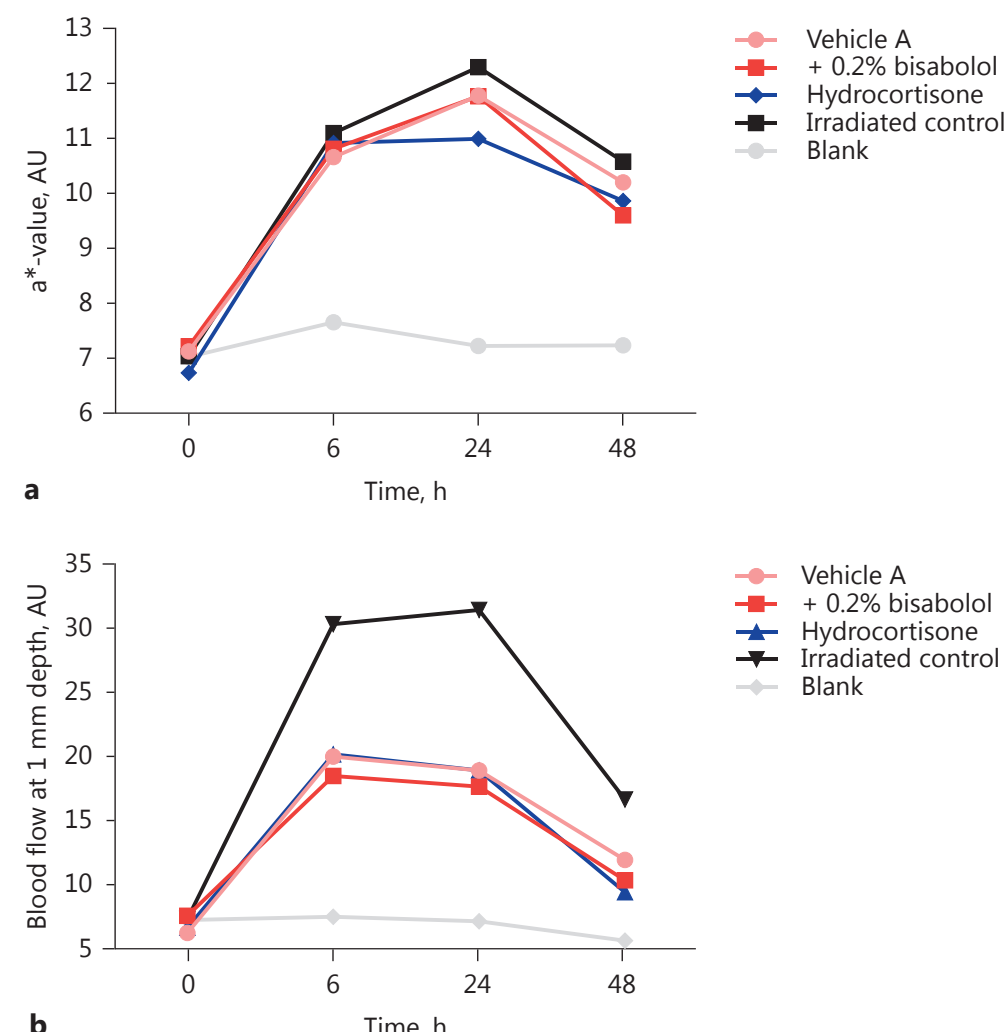

Vehicle A

$+0.2 \%$ bisabolol

Hydrocortisone

$\rightarrow$ Irradiated control

Blank

b

Time, $\mathrm{h}$

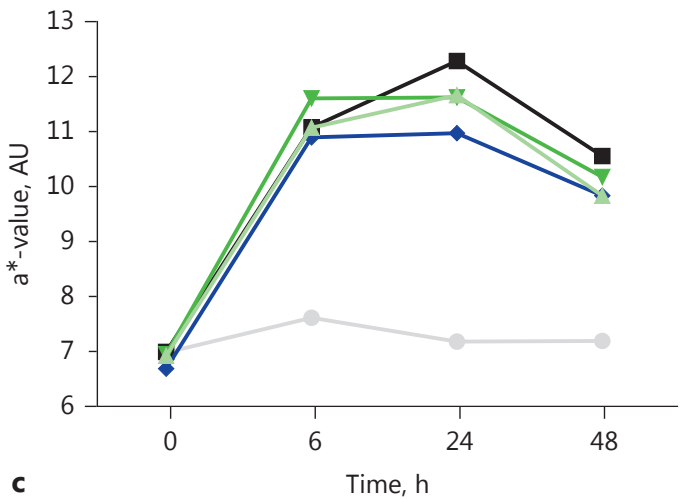

- Vehicle B

$-7+1 \%$ bisabolol

Hydrocortisone

Irradiated control

Blank

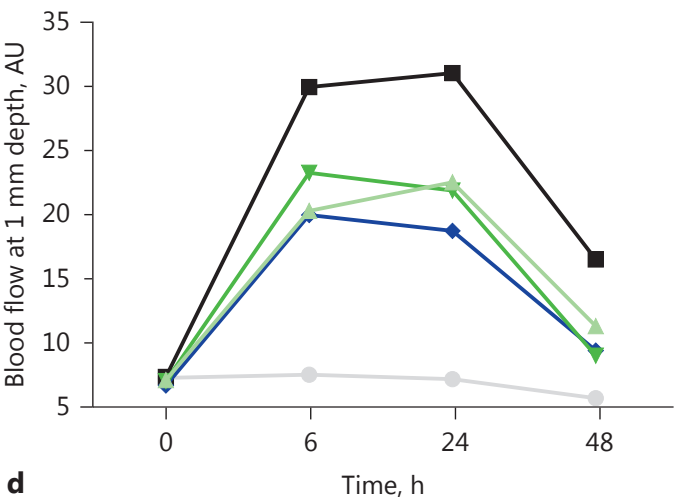

Vehicle B

$+1 \%$ bisabolol

Hydrocortisone

Irradiated control

Blank 


\section{Determination of Antiphlogistic Efficacy}

In the SPF in vivo determination test, the vehicle A formula with $0.2 \%$ bisabolol and the vehicle B formula with $1 \%$ bisabolol deviate the most from the respective vehicle formulas. In order to determine their potentially antiphlogistic efficacy, an erythema model for testing formulas on UV-induced inflammations over a time course of $48 \mathrm{~h}$ was used. The degree of erythema was greatest in the irradiated control field $(\mathrm{AUC}=188)$ and was smallest in the field treated with $1 \%$ hydrocortisone cream $(A U C=149)$. Furthermore, the AUC of the sunscreen vehicle A formula (AUC $=168$ ) was reduced (not significantly) when compared to the irradiated control field $(p=0.2334)$, but not compared to the field treated with $1 \%$ hydrocortisone cream $(p=0.5693)$ or the respective formula with $1 \%$ bisabolol (AUC $=163$; $p=$ 1.0000). The same can be observed for the sunscreen vehicle $\mathrm{B}$ formula $(\mathrm{AUC}=166)$ and the respective formula with $1 \%$ bisabolol $($ AUC $=176)$ (Fig. 2a, c). No statistically significant difference between the vehicle B formula and the formula with $1 \%$ bisabolol could be observed $(p=1.0000)$.

The blood flow at $1 \mathrm{~mm}$ depth showed a significantly reduced AUC with the sunscreen vehicle A formula (AUC $=466)$ when compared to the irradiated control field (AUC $=909 ; p=0.0005)$, but not to the field treated with $1 \%$ hydrocortisone cream (AUC $=439 ; p=$ 0.5566 ) or the respective formula with $1 \%$ bisabolol (AUC $=407 ; p=0.4375)$. However, comparing vehicle A with $0.2 \%$ bisabolol at all time points after treatment, a statistically significant difference at $6 \mathrm{~h}(p=0.0313)$ could be shown.

It can be summarized that the time course of UVinduced erythema (skin reddening and blood flow) is decreased by the sunscreen formulas compared to the irradiated control field. However, the incorporation of bisabolol into the sunscreen formulas showed a similar intensity and time course of UV-induced erythema to that of the vehicle formulas. These results further corroborate the findings from the SPF in vivo determination.

\section{Discussion}

The use of sunscreens benefits health and is one of the measures that dermatologists recommend to reduce photoaging and skin cancer [10]. However, it has been confirmed that certain ingredients in sunscreen products have anti-inflammatory properties and are considered to suppress light-induced erythema, the biological end point for the SPF determination in vivo. When this is the case, the SPF of the sunscreen products is erroneously labeled too high and misleads consumer into exposing themselves excessively to the sun.

To help address this important issue, we analyzed the frequently used ingredients bisabolol and panthenol in sunscreens with regard to their potential to interfere with the development of UV erythema. It is known from the literature that UV filters have anti-inflammatory effects. Diethyhexyl butamido triazone and benzophenone-5, in particular, have substantial anti-inflammatory properties, which have also been reported for homosalate, benzophones, and cinnamic acid derivates [11]. Furthermore, the sunscreen agents oxybenzone and dioxybenzone have been patented as anti-inflammatory agents [12]. These facts, however, do not automatically imply that these filters interfere with light-induced erythema.

Recently, a study was performed by Staton and Feng [13] that determined the anti-inflammatory potential of sunscreen actives in its erythemal regression potential when applied topically to the skin following UV irradiation. These studies were performed in accordance with the EN ISO 24444:2010 standard. It was found that the SPF 100 sunscreen, which contains high levels of actives, did not reduce the erythemal response to UV light.

However, in order to analyze the antiphlogistic effect of bisabolol and panthenol in sunscreens, the prepared formulas must do without sunscreen filters with known anti-inflammatory properties. A first study aimed at providing an answer to this question was performed by Couteau et al. [14], who identified the anti-inflammatory activity of the 3 ingredients allantoin, $\alpha$-bisabolol, and $\beta$ glycyrrhetinic acid incorporated into sunscreens, using a phorbol-myristate-acetate-induced mouse ear model. However, this approach is not applicable since the data obtained were from chemically induced edema and did not involve the UV-induced erythemal response used in the SPF in vivo testing.

In contrast, our study was specifically designed for the purpose of measuring the direct impact of bisabolol and panthenol on the SPF in vivo, using the international standard EN ISO 2444:2010 method. In order to further validate the results we obtained, a UV model with a time course of $48 \mathrm{~h}$ and a repeated application of the formulas after irradiation was used. We found that the incorporation of bisabolol or panthenol into sunscreens did not have an impact on the measured SPF in vivo. Even under 
a higher application rate, no anti-inflammatory effect of the tested antiphlogistics over time could be found.

These findings fit well to the data published by Staton and Feng [13]. The most widely used anti-inflammatory drug, hydrocortisone, incorporated into the ISO P2 (SPF 16) reference sunscreen and applied directly after UV irradiation, showed no statistically significant differences when compared to the $\mathrm{P} 2$ sunscreen.

Based on the data obtained, we can conclude that the incorporated antiphlogistic ingredients panthenol and bisabolol in the tested sunscreen formulas did not interfere with erythema reddening, and thus did not affect the SPF value in vivo. These findings imply, however, that there is no need to incorporate antiphlogistic agents in any quantity into active sunscreens, but that they should rather be used only in after-sun products to alleviate inflammation.

\section{Acknowledgement}

The authors thank Dalli Group GmbH \& Co. KG for formulating the vehicle A (SPF 20) formulas, and Stanislaw Krus from BASF Grenznach GmbH for formulating the vehicle B (SPF 15) formulas. We would like to thank all the volunteers who made this investigation possible.

\section{Statement of Ethics}

The study design for noninvasive surveys was approved by the Ethics Committee of the Witten/Herdecke University, Germany. The subjects read the information sheets and signed the informed consent form prior to study participation.

\section{Disclosure Statement}

The authors report no conflicts of interest in this work.

\section{References}

1 Regulation (EC) No. 1223/2009 of the European Parliament and of the Council of 30 November 2009 on cosmetic products (recast) (text with EEA relevance), Official Journal of the European Union, L 342/59, 22 December 2009.

2 Guidelines to Commission Regulation (EU) No. 655/2013 laying down common criteria for the justification of claims used in relation to cosmetic products (July 2013 version).

3 Cosmetics I: sun protection factor test methods - in vivo determination of SPF (sun protection factor).

4 Maurya AK, Singh M, Dubey V, Srivastava S, Luqman S, Bawankule DU: Alpha-(-)-bisabolol reduces pro-inflammatory cytokine production and ameliorates skin inflammation. Curr Pharm Biotechnol 2014;15:173-181.
5 Haydar K, Burkhart CG: Sunscreen regulations and use of anti-inflammatory agents in sunscreens. Dermatol Online J 2013;19: 18969.

6 Sayre RM: Rapid precision testing laboratory: Citizen Petition FDA, 15 March 2013.

7 Fitzpatrick TB: The validity and practicality of sun-reactive skin types I through VI. Arch Dermatol 1988;124:869-871.

8 Fitzpatrick TB: Soleil et peau. J Med Esthet 1975;2:33-34.

9 Del Bino S, Bernerd F: Variations in skin colour and the biological consequences of ultraviolet radiation exposure. Br J Dermatol 2013; 169(suppl 3):33-40.
10 Hughes MC, Williams GM, Baker P, Green AC: Sunscreen and prevention of skin aging: a randomized trial. Ann Intern Med 2013; 158:781-790.

11 Couteau C, Chauvet C, Paparis E, Coiffard L: UV filters, ingredients with a recognized antiinflammatory effect. PLoS One 2012;7: e46187.

12 Dewhirst F: 2-Hydroxybenzophenones and their use in treating inflammation. US Forsyth Dental Infirmary for Children 1981, vol 4.

13 Staton J, Feng H: Anti-inflammatory effects of sunscreens - wonder or science? Sci Beauty 2015;4:57-61.

14 Couteau C, Chauvet C, Paparis E, Coiffard LJ: Influence of certain ingredients on the SPF determined in vivo. Arch Dermatol Res 2012. 304:817-821. 